\title{
Variación arquitectónica y morfológica de Hintonia latiflora (Rubiaceae) en relación a la cosecha de corteza y factores ambientales
}

\author{
Leonardo Beltrán-Rodríguez, Angélica Romero-Manzanares, Mario Luna-Cavazos* \\ \& Edmundo García-Moya \\ Postgrado en Botánica, Colegio de Postgraduados, Carretera México-Texcoco, Montecillo, Texcoco, Edo. de México, \\ 56230, México; beltran.leonardo@colpos.mx,dahly@colpos.mx,mluna@colpos.mx, edmundo@colpos.mx \\ * Correspondencia
}

Recibido 06-VII-2016. Corregido 12-IV-2017. Aceptado 16-V-2017.

\begin{abstract}
Architectural and morphological variation of Hintonia latiflora (Rubiaceae) in relation to bark harvest and environmental factors. Hintonia latiflora is a rare ecologically and economically important species from the Tropical Deciduous Forest of the Balsas Basin, Mexico, whose bark is traded as medicinal. Debarking practices have modified the shape of plants, their architecture and morphometry; but it is unknown if some topographic and edaphic factors may also influence in these attributes. Here we propose that the ecological conditions of this species distribution and harvesting areas, may determine the morphometric characteristics and the individuals variation in their architecture. To test this hypothesis we assessed the relationship of topographic and edaphic factors on the morphological characteristics of saplings $(\mathrm{n}=143)$ and adults $(\mathrm{n}=117)$, in eight harvesting areas of H. latiflora. The harvesting areas, with a surface of 0.6 ha, were selected randomly with the support of participatory mapping elaborated by gatherers of the study site. Data were analyzed using principal components (PCA) and canonical correspondence (CCA) analyses. The first three PC explained $92.1 \%$ of the morphological variation; height, basal diameter, and coverage, explained morphometric differences in both, saplings and adults, in all eight harvesting zones. The terrain slope, slope orientation, soil depth and stoniness, were ecological factors statistically related with morphometric differences. A lower terrain slope, little stoniness, soil depth, slope orientation E-W in saplings (azimuth $90^{\circ}-270^{\circ}$ ) and $\mathrm{N}-\mathrm{S}$ in adults (azimuth $0^{\circ}-180^{\circ}$ ) were the factors associated to height, number of basal branches, basal diameter and coverage of $H$. latiflora. Areas with higher harvesting intensity were composed by monopodic trees and some reiterated basitone individuals; the adults of these areas also had more fruits. At these sites, plants of H.latiflora were more abundant and produced more bark, but the high-intensity of harvesting, changes plant architecture, forming shrubby, multi-stemmed individuals, that originally were mesotone trees. Areas with lower harvesting intensity were characterized by steep slopes, shallow and rocky soils, and were dominated by basitone trees of smaller size, thin and scarce in the area. These sites are the least recommended for harvesting, because they produce less bark per tree, and serve to preserve the species at the site. Based on these results, we can develop restoration programs in areas affected by commercial harvesting, establish forestry plantations in key sites to ensure the establishment and development of individuals with desirable characteristics for bark harvesting and contribute to in situ conservation of H. latiflora in the Alto Balsas Basin. Rev. Biol. Trop. 65 (3): 900-916. Epub 2017 September 01.
\end{abstract}

Key words: commercial harvesting, medicinal bark, plant morphometric, plant architecture, tropical deciduous forest.

La arquitectura vegetal consiste en el arreglo de la biomasa en el espacio mediante patrones de ramificación (Hallé, 2010), a través del equilibrio entre procesos de crecimiento endógenos y restricciones exógenas mediadas por factores ambientales (Barthélémy \& Caraglio,
2007). La variación morfológica entre individuos de la misma especie es el resultado de los factores físicos del medio, tales como la luz, inclinación de la pendiente, cantidad de precipitación y tipo de suelo (Ouinsavi \& Sokpon, 2010). Por ejemplo, Pearcy, Muraoka 
y Valladares (2005) mencionaron que la intensidad lumínica influye en el crecimiento y desarrollo de especies de Psychotria, a través de la relación longitud de entrenudos, cambio en los ángulos foliares, eficiencia en la captura de luz y ganancia de carbono. En árboles de los bosques estacionalmente secos se observó mayor estatura y longevidad asociada con la disponibilidad de luz (Poorter, Bongers, \& Bongers, 2006). En otro estudio, se encontró que la diferente radiación solar recibida en las laderas norte y sur durante el día y a través del año, influyó en la densidad, número y disposición de ramas de individuos de Neobuxbaumia tetetzo (J.M. Coult.) Backeb. (López-Gómez, Zedillo-Avelleyra, Anaya-Hong, GonzálezLozada, \& Cano-Santana, 2012). En la selva húmeda de México, Martínez-Sánchez, Meave y Bongers (2008) encontraron que la arquitectura de la copa de árboles juveniles tolerantes a la sombra, de los géneros Guamia sp., Trophis sp. y Pseudolmedia sp., cambió de acuerdo con la disponibilidad de luz y al estrato que ocupan las especies en un perfil vertical de la vegetación.

El factor edáfico, la pendiente y la precipitación, influyeron en el crecimiento y alometría en individuos de Milicia excelsa (Welw.) C.C. Berg., especie de los bosques tropicales húmedos del África Subsahariana (Ouinsavi \& Sokpon, 2010). Para la Selva Baja Caducifolia de Yucatán, México, Interián-Ku et al. (2009) mencionan que el suelo, tipo luvisol versus leptosol, incidió en la arquitectura arbórea de Caesalpinia gaumeri Greenm. y Gymnopodium floribundum Rolfe, especialmente debido a cambios en el grosor del tallo y en el ángulo de inserción de ramas de segundo y tercer orden.

En relación con la arquitectura arbórea entre estadios de desarrollo, Pineda-Herrera, Valdez-Hernández, López-López, SalgadoUgarte y Manzano-Méndez (2011) analizaron la arquitectura de Schizolobium parahyba (Vell.) S. F. Blake y Vochysia guatemalensis Donn. Sm., dos especies de la selva alta perennifolia de Oaxaca, México, y encontraron que los brinzales (plántulas) y latizales (juveniles) se ajustaron a diferentes modelos arquitectónicos
(Cook y Massart, respectivamente; sensu Hallé, Oldemann y Tomlinson, 1978)) con respecto a los fustales (adultos; Leeuwenberg y Scarrone, respectivamente), con base en diferencias morfométricas en variables como altura total, diámetro a la altura del pecho, longitud y ángulo de ramas de primer orden y promedio de copa.

En Latinoamérica, faltan estudios que analicen el efecto del aprovechamiento no maderable como factor influyente en la arquitectura de especies arbóreas tropicales de uso comercial (Alexiades \& Shanley, 2004). A la fecha, sólo existe información de tipo demográfico para manejo forestal como en el caso de Bursera glabrifolia (Kunth) Engl. (Hernández-Apolinar, Valverde, \& Purata, 2006), y otras especies (38 a 50) incluidas en estudios de cambio en la diversidad y de los patrones de dominancia o de invasión de especies por efecto de cosecha (Rendón-Carmona, Martínez-Yrízar, Balvanera, \& Pérez-Salicrup, 2009). En África, en cambio, se ha documentado el contexto económico internacional, la situación social de los recolectores y estudios de caso sobre la ecología y sostenibilidad de cosecha de corteza en 20 especies (Cunningham, Campbell, \& Luckert, 2014).

A pesar de que México es uno de los países latinoamericanos en donde más se aprovechan especies forestales no maderables con fines comerciales (Alexiades \& Shanley, 2004; Stanley, Voeks, \& Short, 2012), existe poca información sobre la influencia de factores ecológicos y de manejo en la arquitectura y morfometría de las especies, y más aún, entre diferentes estadios de desarrollo (i.e. plántula, juvenil y adulto), lo que limita la aplicación de prácticas silvícolas basadas en el conocimiento de las condiciones de sitio.

El concepto de modelo arquitectónico fue inicialmente trabajado en especies tropicales por Hallé, Oldemann y Tomlinson (1978), quienes a partir de un amplio inventario forestal redujeron la diversidad arbórea a 23 modelos arquitectónicos. Las características incluidas en cada modelo fueron detalles morfológicos identificados de manera descriptiva: la forma de ramificación, su distribución sobre el tronco, 
el desarrollo de las yemas axilares por silepsis (si no tienen letargo, crecimiento continuo) o por prolepsis (si hay letargo, crecimiento rítmico), la extensión de los nuevos vástagos, la diferenciación de ramas en ortotrópicas (erectas y con simetría radial) o plagiotrópicas (horizontales con simetría dorsiventral), la posición de las flores en yemas laterales o terminales y la orientación del dosel. El modelo arquitectónico resume la forma en términos cualitativos.

Hintonia latiflora (Sessé \& Moc. ex DC.) Bullock por sus características pertenece al Modelo Leeuwenberg (Beltrán-Rodríguez et al., 2015). Es un árbol con tallo monopodial, ramificación mesótona (inicia a la mitad de la longitud del tallo), posee ramas ortotrópicas y crecimiento determinado con inflorescencias en posición terminal. La ramificación produce varios ejes equivalentes determinados por inflorescencia en el caso de los adultos, y por disfuncionalidad del ápice en el caso de los juveniles (Vester, 2002). Algunas plantas presentan reiteraciones, es decir, repeticiones del modelo original en ramas, una vez que las plantas han sufrido alguna alteración como podas, cosecha, rompimientos de ramas o algún otro trauma. Hallé (2010) describe a la reiteración como la formación de un árbol pequeño pero completo sobre un tronco o una rama. La importancia biológica de este modelo arquitectónico es que por el porte, H. latiflora representa un elemento arbóreo distintivo dentro de la comunidad de la Selva Baja Caducifolia, sus inflorescencias apicales facilitan la dispersión de sus frutos por anemocoria, las ramas ortotrópicas dejan a la vista el tronco que por longitud y grosor, facilita la cosecha de corteza en grandes fracciones longitudinales.

Hintonia latiflora conocida como "quina amarilla”, es una planta con importancia económica nacional e internacional por la cosecha y comercialización de la corteza extraída exclusivamente de poblaciones silvestres procedentes de la Cuenca del Balsas, México. Actualmente, la quina amarilla tiene 36 usos en la medicina tradicional mexicana, el antidiabético y febrífugo son los más importantes (Mata, Navarrete, Cristians, Hersch-Martínez, \& Bye, 2009). El precio de venta promedio de la corteza en seco es de $\$ 57$ pesos $\mathrm{kg}^{-1}$ (\$ 3 USD) en los mercados principales de la Ciudad de México. En Europa, existe una industria creciente de extractos medicinales elaborados con $H$. latiflora exportada ilegalmente (Hersch-Martínez, 2007), vendidos en \$ 53 USD promedio ( \pm 40 USD). El margen de ganancia del recolector es de $\$ 10$ pesos $\mathrm{kg}^{-1}$ (\$0.53 USD). Sin embargo, la alta demanda de corteza contribuye a la sobreexplotación de este recurso (Beltrán-Rodríguez et al., 2015). Y a pesar de la importancia medicinal y económica de esta especie, se carece de estudios que permitan establecer criterios para su aprovechamiento y conservación.

La constante cosecha de corteza y las malas prácticas durante el descortezado, como la derriba en pie y cosecha de ramas, han modificado la forma de las plantas de quina amarilla (Beltrán-Rodríguez et al., 2015) así como el patrón arquitectónico (modelo bidimensional de la forma de la planta) y la morfometría (la medida de las partes estructurales). La forma en que se cosecha la corteza a lo largo del fuste y de las ramas de primer y segundo orden, reduce las existencias reales de corteza aprovechable en poblaciones silvestres, lo que induce al descortezamiento de individuos con dimensiones alométricas no comerciales.

Evaluamos siete variables topográficas y edáficas, además de diferentes intensidades de cosecha de corteza con el objetivo de determinar si las condiciones ecológicas en los sitios de distribución y cosecha modifican la arquitectura e influyen en la variación de algunos caracteres morfológicos de Hintonia latiflora, tanto en individuos juveniles como adultos.

Debido a que la quina amarilla se distribuye en regiones montañosas de la Cuenca del Alto Balsas, México, la hipótesis asume que las condiciones ecológicas en los sitios de distribución natural así como de cosecha, determinarán la variación del modelo arquitectónico, la variación cuantitativa de las características morfológicas, y la distinción por morfometría de plantas adultas y juveniles. Dada la importancia económica de $H$. latiflora en México, la información que genere este estudio sobre 
la relación forma de la planta y variables ambientales, será potencialmente importante para saber bajo cuáles circunstancias ambientales encontrar a la especie, determinar cuáles son algunas de las mejores condiciones para el desarrollo morfométrico y utilizar a la especie bajo esos criterios, para rehabilitar sitios degradados o sobrecosechados.

\section{MATERIALES Y MÉTODOS}

Especie de estudio: Hintonia latiflora es una planta de porte arbustivo a arbóreo, perenne, iterópara y hermafrodita, de 15-20 $\mathrm{cm}$ en diámetro a la altura de la base, $6-8 \mathrm{~m}$ de altura y ancho de copa de 3.5-6.5 m (Ochoterena, 2000), reproductiva a partir de $8 \mathrm{~cm}$ de diámetro basal (Beltrán-Rodríguez et al., 2015). Es una especie endémica del bosque tropical caducifolio con distribución geográfica en México y Centroamérica (Lorence \& Taylor, 2011), pero con poblaciones aisladas y baja densidad de individuos reproductivos (5 ind. $\mathrm{ha}^{-1}$ ), restringidos a laderas y cañadas (BeltránRodríguez et al., 2015). La historia de recolección de corteza de Hintonia latiflora con fines comerciales data de hace 40 años en la Cuenca del Balsas, México (Hersch-Martínez, Fierro, Guerrero, \& Pérez, 2000; Hersch-Martínez \& Fierro, 2001), sin regulación oficial por el gobierno mexicano, pero normada localmente por autoridades comunitarias.

Sitio de trabajo: La investigación se llevó a cabo durante el periodo enero-diciembre 2012 en la Cuenca del Balsas, específicamente en la porción conocida como Alto Balsas, del

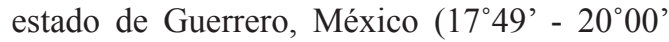
$\mathrm{N} \& 99^{\circ} 03^{\prime}-99^{\circ} 13^{\prime} \mathrm{W}$ ); área de distribución natural y de cosecha comercial de corteza en poblaciones silvestres de la quina amarilla. El clima según la clasificación de Köppen adaptada a la República Mexicana es $\mathrm{BS}_{1}\left(\mathrm{~h}^{\prime}\right) \mathrm{w}(\mathrm{w})$ ig, seco y semicálido, con lluvias de verano, $800 \mathrm{~mm}$ de precipitación promedio anual y temperatura anual promedio de $18{ }^{\circ} \mathrm{C}$ (García, 1988). El relieve consiste de montañas y cerros con pendientes abruptas $\left(\sim 50-60^{\circ}\right)$, entre cotas altitudinales de 560 y $1580 \mathrm{~m}$. El material parental es sedimentario, presente a menos de $15 \mathrm{~cm}$ de profundidad, suelos tipo Leptosol, arcillosos y someros, y Rendzina, rojos, pobres en materia orgánica, pedregosos, bien drenados y susceptibles a la erosión (INEGI, 2009). El área es un mosaico de vegetación con campos agrícolas y bosques secundarios en las planicies próximas al Río Balsas, y con bosques primarios relativamente bien conservados en las zonas montañosas más alejadas. La vegetación dominante es Bosque Tropical Caducifolio con predominio de las familias Fabaceae, Burseraceae y Poaceae, pero también hay Matorral Xerófilo tipificado por cactáceas columnares.

Zonas de cosecha: Se seleccionaron ocho zonas (Z1 a Z8) diferentes en intensidad de cosecha de corteza, agrupadas en dos niveles: alta $(n=4)$ y baja $(n=4)$ intensidad; no existieron poblaciones sin cosechar (Cuadro 1). La intensidad de cosecha se basó en el número de individuos descortezados registrados por zona, $\leq 10 \%$ de la población total se consideró como baja intensidad $\mathrm{y} \geq 10.1 \%$ fue alta intensidad. Cada zona de cosecha tuvo una superficie de 0.6 ha y una separación espacial variable, generalmente mayor a $1000 \mathrm{~m}$. Estas poblaciones fueron elegidas al azar (de un total de 17 zonas de cosecha) mediante previos mapeos participativos confeccionados con recolectores. Para medir diferencias estadísticas entre zonas de cosecha por la densidad de individuos descortezados por estadio de desarrollo, se aplicó un análisis de varianza (ANAVA) y una prueba de comparación de medias de Tukey $(\alpha=0.01)$ por medio del programa estadístico InfoStat versión 2013 (Di Rienzo et al., 2013).

Factores ecológicos: En cada zona de cosecha se registraron siete factores ecológicos: 1) altitud en msnm, tomada con un sistema de geoposicionamiento global (GPS MAP 62SC, Garmin, México); 2) orientación de la ladera en grados azimut, a partir del ángulo generado entre el punto Norte de referencia y el sitio de estudio medido con brújula; 3 ) pendiente del terreno, en grados, medida con clinómetro 


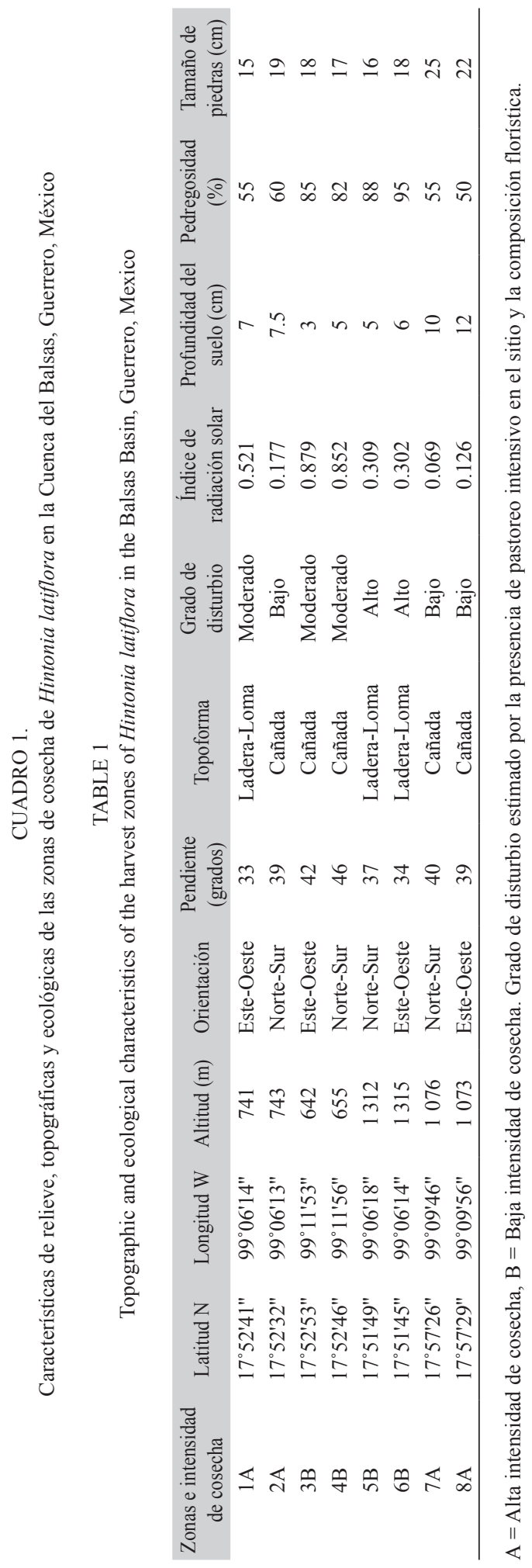

(Kode KB-14/360, Suunto, USA); 4) índice de radiación solar, calculada con el algoritmo propuesto por Oke (1987) basado en pendiente y azimut; 5) profundidad del suelo en $\mathrm{cm}$, retirando primero la capa de hojarasca, a tres niveles: superficial $0.1-5 \mathrm{~cm}$, poco profundo $5.1-10 \mathrm{~cm}$ y profundo $\geq 10.1 \mathrm{~cm} ; 6$ ) pedregosidad estimada por categorías porcentuales $(0-25 \%, 26-50$ $\%, 51-75 \%, 76-100 \%)$ y, 7) tamaño de piedras por diámetro de partículas, y categorizado $(<5$ $\mathrm{cm}, 5-10 \mathrm{~cm}, 10-20 \mathrm{~cm} \mathrm{y} \geq 20 \mathrm{~cm}$ ) (Cuadro 1).

Muestreo de juveniles y adultos de $\boldsymbol{H}$. latiflora: En cada zona de cosecha se establecieron tres unidades de muestreo (UM) de $20 \mathrm{x}$ $100 \mathrm{~m}$, separadas entre sí cada $25 \mathrm{~m}$. Las UM fueron rectángulos en los que se cuantificó la densidad de la especie. El recuento y medición de plantas adultas se llevó a cabo en la superficie total de las $24 \mathrm{UM}$, mientras que para cuantificar y medir plantas juveniles esos rectángulos fueron divididos en 96 subunidades de muestreo de $8 \times 20 \mathrm{~m}$ (cuatro subunidades por rectángulo). Los juveniles fueron individuos no reproductivos (sin evidencias de haberse reproducido) con altura $\geq 10.1 \mathrm{~cm} \leq 3 \mathrm{~m}$ y diámetro basal $\geq 0.6 \mathrm{~cm} \leq 8 \mathrm{~cm}$, mientras los adultos fueron plantas reproductivas (florecieron y fructificaron), con altura $\geq 3.1 \mathrm{~m} \leq 8 \mathrm{~m}$ y diámetro basal $\geq 8.1 \mathrm{~cm} \leq 20 \mathrm{~cm}$.

Arquitectura: Se determinó con base en los morfotipos consignados por Beltrán-Rodríguez et al. (2015). Las plantas de H. latiflora siguen el modelo arquitectónico Leeuwenberg, pero evidencian variaciones: en su ámbito natural presentan porte monopódico mesótono (un tallo que ramifica desde la mitad del largo del fuste hacia la parte más distal de la copa) y, en la forma reiterada, son plantas de porte arbustivo multirramificado basítono (tallo ramificado desde la base). Para cada variante arquitectónica, se distinguió en campo si se trataba de individuos juveniles o adultos. Para determinar si existían diferencias significativas en el número de individuos monopódicos y multirramificados por estadios de desarrollo y entre zonas de cosecha, se aplicó un ANAVA y una prueba 
de comparación de medias de Tukey $(\alpha=0.01)$, mediante el programa estadístico InfoStat versión 2013 (Di Rienzo et al., 2013).

\section{Diferenciación morfométrica de juveni-} les y adultos por zona de cosecha: En cada uno de los individuos juveniles y adultos de $H$. latiflora previamente censados, se registraron siete variables que incluían caracteres morfométricos y reproductivos: altura total, diámetro basal, amplitud de la cobertura estimada por dos diámetros tomados de manera perpendicular, número de ramificaciones basales, ángulo de inclinación de las ramas basales con respecto al suelo, número de órdenes de ramificación $\mathrm{y}$, en el caso de los adultos, número total de frutos por planta.

Los caracteres morfológicos medidos de los individuos juveniles y adultos de $H$. latiflora se analizaron tanto de manera conjunta como de forma independiente, debido a que ambos estadios de desarrollo son de interés para la cosecha y que la producción de corteza entre estadios podría ser variable. Para esto, se elaboró una matriz de datos con los individuos juveniles y adultos registrados en cada unidad de muestreo (UM) de las ocho zonas de cosecha; en total se registraron 143 individuos juveniles y 117 adultos para todas las unidades de muestreo. Se aplicó un análisis de componentes principales (ACP) para examinar el patrón de diferenciación de los individuos por morfometría; en tal caso, se consideró el promedio de las variables registradas en los individuos ya sea de la etapa juvenil o adulta de cada UM. Las UM donde no se registraron individuos adultos o juveniles fueron excluidas del análisis. Luego, mediante una Prueba de t para dos muestras se compararon los valores resultantes del ACP de adultos y juveniles, para determinar si existen diferencias significativas entre ambos estadios de desarrollo de $H$. latiflora .

Adicionalmente, se efectuó un ANAVA no paramétrico con la prueba de Kruskal-Wallis de los caracteres morfológicos, tanto de individuos juveniles y adultos; y comparaciones por pares entre las medias de las poblaciones para estimar diferencias significativas entre ellas $(\alpha=0.05)$; se eligió esta prueba dado que el número de individuos fue reducido (3) en algunas zonas. Este análisis se efectuó con el programa estadístico InfoStat versión 2013 (Di Rienzo et al., 2013).

Influencia de los factores ecológicos de las zonas de cosecha sobre la morfometría de Hintonia latiflora: Un análisis de correspondencia canónica (ACC) fue usado para evaluar la relación entre los factores ecológicos de las zonas de cosecha con las características morfológicas medidas en las poblaciones de H. latiflora. Previo a esto, se seleccionaron los factores ecológicos que diferencian las ocho zonas de cosecha mediante un ACP y, con base en los mayores valores de carga, el análisis seleccionó a la orientación de la ladera, el tamaño de piedras, la profundidad del suelo y la pendiente, como los factores a considerar para el ACC. Debe ser mencionado que el registro de las variables ambientales se efectuó por zona de cosecha; para este análisis se elaboraron dos matrices de datos, la primera con ocho poblaciones de $H$. latiflora (Z1 a Z8) y siete variables morfométricas y, la segunda, con las cuatro variables ecológicas mencionadas que caracterizan a las zonas de cosecha donde se encontraron las poblaciones. Los análisis de componentes principales, y correspondencia canónica se efectuaron con el programa PCORD versión 6.0. (McCune \& Mefford, 2011).

\section{RESULTADOS}

Zonas de cosecha y el aprovechamiento de plantas juveniles y adultas: Las zonas de cosecha se ubicaron en altitudes de 741 a $1073 \mathrm{msnm}$, cuatro de estas con orientación norte-sur y el resto con dirección este-oeste; pendientes ligeramente pronunciadas en laderas y cañadas. Las zonas de cosecha presentan disturbio de bajo a moderado, a veces alto; suelos someros entre 3 a $12 \mathrm{~cm}$, promedio de $7 \mathrm{~cm}$ y altamente pedregosos (Cuadro 1). Se encontraron diferencias significativas entre zonas de acuerdo con la intensidad de cosecha de corteza $(\mathrm{F}=27.22, \mathrm{P}=0.0020$, juveniles; 
$\mathrm{F}=17.50, \mathrm{P}=0.0058$, adultos). En las áreas de muestreo, el descortezamiento para aprovechamiento fue mayor en individuos adultos respecto a los juveniles; existió un alto porcentaje de individuos juveniles no descortezados. La densidad de la quina amarilla en la región muestreada (4.8 ha) del Alto Balsas fue de 260 individuos, en proporciones cercanas al $50 \%$ entre juveniles y adultos (Cuadro 2).

Arquitectura de plantas juveniles y adultas de quina amarilla: El modelo arquitectónico entre individuos juveniles y adultos no mostró variación. Pero ambos estadios de desarrollo presentaron dos diferentes morfotipos, monopódico y multirramificado. Las plantas juveniles tienen un comportamiento principalmente monopodial $(\mathrm{n}=109)$, mientras los adultos tienden a multirramificarse de base $(\mathrm{n}=72)$. No se encontraron diferencias significativas $(\mathrm{P}<0.01)$ en el número de individuos monopódicos y multirramificados en zonas con diferente intensidad de cosecha, tanto en juveniles $(\mathrm{F}=5.32, \mathrm{P}=0.0369)$ como en adultos $(\mathrm{F}$ $=0.32, \mathrm{P}=0.5793$ ) (Cuadro 2).

Variación de los caracteres morfométricos en individuos juveniles y adultos de Hintonia latiflora: El análisis de ordenación por componentes principales (CP), para juveniles y adultos, explicó el $92.1 \%$ de la variación, y diferencia ampliamente a las poblaciones juveniles y adultas. En el CP1, que explica el 58.9\% de la variación, las variables con mayores factores de carga fueron la cobertura medida como diámetro1 y diámetro 2, la altura de planta, el número de órdenes de ramificación y el DAB (Cuadro 3). En la gráfica derivada de este análisis, se observa que hacia el lado izquierdo de la misma se ordenan los individuos con mayor cobertura de copa, más altos, mayor número de órdenes de ramificación y DAB, que corresponde con individuos adultos, a diferencia de los juveniles ubicados en el extremo opuesto. El CP2 contiene el $20.9 \%$ de la variación y el factor de carga más relevante corresponde al número de ramas, y básicamente permite observar que los individuos adultos de los sitios
1, 2 y 3 presentan mayor número de ramas que el resto (Fig. 1). La Prueba de t demostró diferencias altamente significativas en el caso de los valores resultantes del componente principal uno $(T=-9.86, p=0.0001)$, pero no para el componente dos $(\mathrm{T}=1.04, \mathrm{p}=0.3071)$.

El análisis de varianza para caracteres morfológicos mostró que, en el caso de juveniles, con excepción del ángulo de inclinación de las ramas, el resto de las características presentaron diferencias significativas. Los contrastes más evidentes ocurrieron en el diámetro a la altura de la base, diámetro 1 y 2 , donde los individuos de las zonas 2,7 y 8 se diferenciaron por presentar los mayores valores promedio de estas estructuras con respecto a las otras zonas. Asimismo, presentaron en general una mayor altura y, en el caso de las zonas 1 y 2, mayor número de ramas basales y órdenes de ramificación (Cuadro 4).

Respecto a los individuos adultos, no hubo diferencias significativas en altura entre las diferentes zonas. En las zonas 1 y 2 se encontraron los individuos con mayor diámetro a la altura de la base y, en estas, al igual que las zonas 6,7 y 8 los mayores valores promedio de diámetro 1 y 2 . En cuanto a las ramas basales, las zonas 1 y 2 se diferenciaron de las otras zonas; mientras que en el caso del ángulo de inclinación de las ramas y órdenes de ramificación mostraron diferencias principalmente entre la zona 5 y el resto (Cuadro 4).

Influencia de los factores ecológicos sobre la variación morfométrica de Hintonia latiflora: El análisis de correspondencia canónica-ACC indicó la influencia de las variables ecológicas en la variación morfológica de las poblaciones de $H$. latiflora. En el caso de los individuos juveniles se obtuvieron correlaciones entre factores ecológicos-variables morfométricas de 0.99 en el eje 1 y de 0.97 en el eje 2 , estadísticamente significativas $(\mathrm{P}<0.01)$. Ambos ejes explicaron en conjunto el $88.7 \%$ de la variación (Cuadro 5).

Con base en la dirección e influencia de los factores ecológicos, la variación morfométrica de individuos juveniles registrada en las 


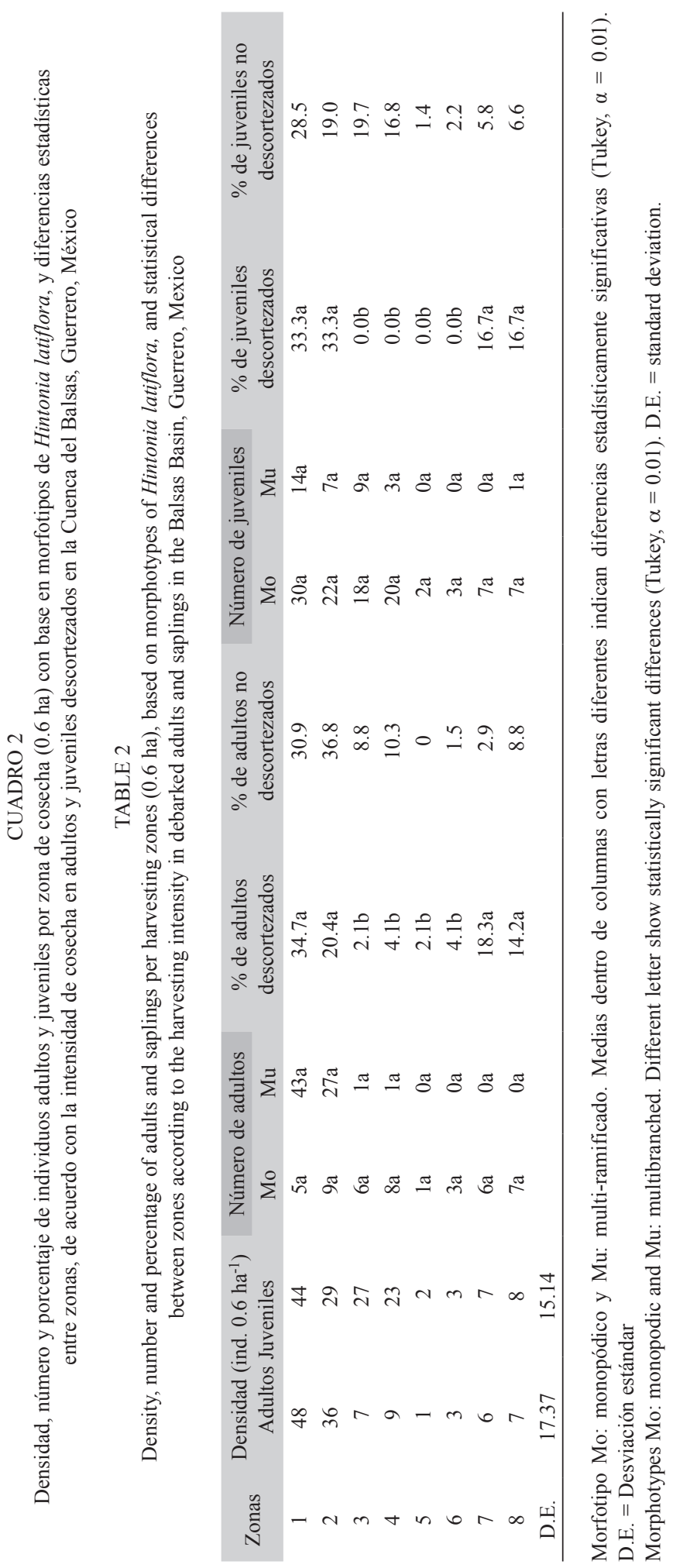




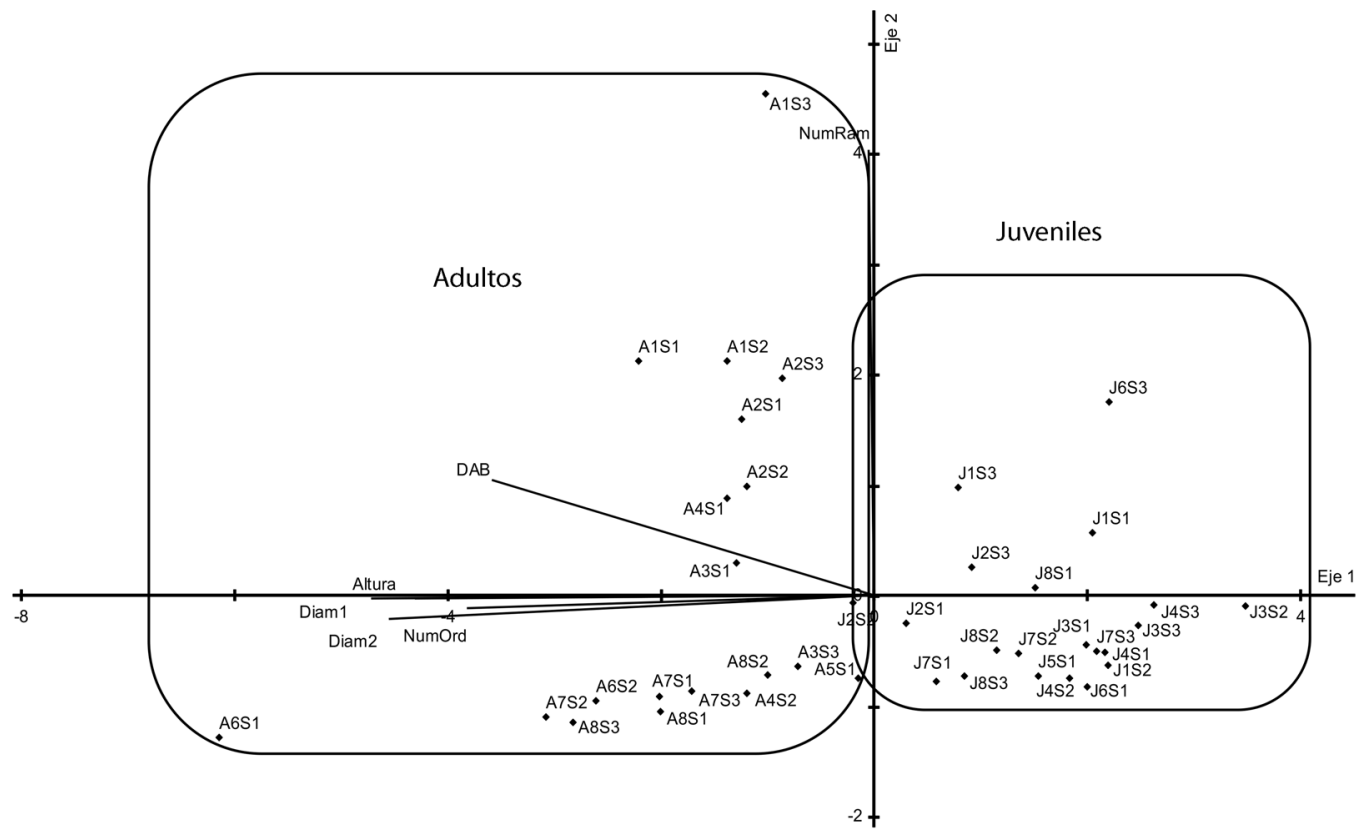

Fig. 1. Patrones de variación morfológica de individuos juveniles y adultos de Hintonia latiflora en el espacio de los componentes principales uno y dos. A1-8 = adultos de las zonas 1 a $8, \mathrm{~J} 1-8=$ juveniles de las zonas 1 a $8, \mathrm{~S} 1-3=$ unidades de muestreo. DAB = diámetro basal, Diam 1 = diámetro 1, Diam 2 = diámetro 2, NumOrd = número de ordenes de ramificación. Fig. 1. Patterns of morphological variation of sapling and adults of Hintonia latiflora in the space of the principal components one and two. A1-8 = adults of the zones 1 to $8, \mathrm{~J} 1-8=$ saplings of the zones 1 to $8, \mathrm{~S} 1-3=$ sampling units. DAB $=$ diameter at the base, Diam 1, $2=$ diameter 1 and 2, NumOrd = number of branching orders.

\section{CUADRO 3}

Valores y vectores característicos del análisis de componentes principales de los caracteres morfológicos de las poblaciones adultas y juveniles de Hintonia latiflora

TABLE 3

Eigenvalues and eigenvectors of the principal component analysis to all sapling and adult populations of Hintonia latiflora

\begin{tabular}{lccc} 
& CP1 & CP2 & CP3 \\
Valores característicos & 4.124 & 1.464 & 0.863 \\
Variación explicada (\%) & 58.9 & 20.9 & 12.3 \\
& & & \\
Factores de carga & & & \\
Altura & -0.918 & -0.077 & -0.179 \\
Diámetro basal & -0.838 & 0.452 & 0.202 \\
Diámetro 1 & -0.962 & -0.082 & 0.004 \\
Diámetro 2 & -0.945 & -0.204 & 0.033 \\
Número de ramas & -0.098 & 0.888 & 0.421 \\
Ángulo de inclinación & 0.016 & -0.624 & 0.777 \\
Ordenes de ramificación & -0.864 & -0.154 & -0.081 \\
\hline
\end{tabular}


CUADRO 4.

Variación de los caracteres morfológicos de individuos juveniles y adultos entre zonas de cosecha de H. latiflora de la Cuenca del Balsas, Guerrero, México

TABLE 4

Morphological variation of saplings and adults individuals among harvest zones in the Balsas Basin, Guerrero, Mexico

Juveniles

\begin{tabular}{llllllll} 
& Altura & DAB & Diam 1 & Diam 2 & RamBas & AngInc & OrdRam \\
Z1 & $2.11 \mathrm{bc}$ & $4.04 \mathrm{a}$ & $109.98 \mathrm{a}$ & $118.89 \mathrm{ab}$ & $1.98 \mathrm{c}$ & $87.02 \mathrm{a}$ & $3.86 \mathrm{~cd}$ \\
$\mathrm{Z} 2$ & $2.96 \mathrm{c}$ & $5.38 \mathrm{~b}$ & $197.97 \mathrm{~b}$ & $168.69 \mathrm{~b}$ & $1.48 \mathrm{bc}$ & $80.34 \mathrm{a}$ & $4.24 \mathrm{~d}$ \\
$\mathrm{Z3}$ & $1.28 \mathrm{a}$ & $2.78 \mathrm{a}$ & $64.31 \mathrm{a}$ & $66.22 \mathrm{a}$ & $1.44 \mathrm{bc}$ & $90.00 \mathrm{a}$ & $3.04 \mathrm{ab}$ \\
$\mathrm{Z} 4$ & $1.94 \mathrm{ab}$ & $3.14 \mathrm{a}$ & $98.74 \mathrm{a}$ & $92.78 \mathrm{a}$ & $1.22 \mathrm{abc}$ & $90.00 \mathrm{a}$ & $3.70 \mathrm{bcd}$ \\
$\mathrm{Z} 5$ & $1.71 \mathrm{ab}$ & $1.83 \mathrm{a}$ & $59.75 \mathrm{a}$ & $63.25 \mathrm{a}$ & $0.50 \mathrm{a}$ & $45.00 \mathrm{a}$ & $1.50 \mathrm{a}$ \\
$\mathrm{Z6}$ & $2.04 \mathrm{abc}$ & $2.33 \mathrm{a}$ & $60.00 \mathrm{a}$ & $67.50 \mathrm{a}$ & $0.75 \mathrm{ab}$ & $45.00 \mathrm{a}$ & $2.25 \mathrm{ab}$ \\
$\mathrm{Z7}$ & $2.08 \mathrm{abc}$ & $5.99 \mathrm{~b}$ & $198.13 \mathrm{~b}$ & $199.50 \mathrm{~b}$ & $1.00 \mathrm{ab}$ & $90.00 \mathrm{a}$ & $3.13 \mathrm{abc}$ \\
$\mathrm{Z} 8$ & $2.10 \mathrm{abc}$ & $6.46 \mathrm{~b}$ & $190.89 \mathrm{~b}$ & $181.44 \mathrm{~b}$ & $1.11 \mathrm{abc}$ & $86.89 \mathrm{a}$ & $3.33 \mathrm{abc}$ \\
\hline
\end{tabular}

Adultos

\begin{tabular}{lllllllll} 
& Altura & DAB & Diam 1 & Diam 2 & RamBas & AngInc & OrdRam & NumFru \\
Z1 & $3.75 \mathrm{a}$ & $17.51 \mathrm{c}$ & $286.44 \mathrm{c}$ & $298.06 \mathrm{bc}$ & $3.33 \mathrm{~b}$ & $83.65 \mathrm{ab}$ & $4.77 \mathrm{bc}$ & $449.50 \mathrm{c}$ \\
$\mathrm{Z} 2$ & $3.59 \mathrm{a}$ & $12.63 \mathrm{~b}$ & $280.17 \mathrm{c}$ & $265.89 \mathrm{ab}$ & $2.47 \mathrm{~b}$ & $74.56 \mathrm{ab}$ & $4.53 \mathrm{ab}$ & $180.22 \mathrm{~b}$ \\
$\mathrm{Z3}$ & $3.38 \mathrm{a}$ & $9.40 \mathrm{ab}$ & $173.08 \mathrm{ab}$ & $164.16 \mathrm{a}$ & $1.13 \mathrm{a}$ & $78.75 \mathrm{ab}$ & $5.25 \mathrm{c}$ & $4.50 \mathrm{a}$ \\
$\mathrm{Z} 4$ & $3.41 \mathrm{a}$ & $8.82 \mathrm{a}$ & $257.90 \mathrm{bc}$ & $221.00 \mathrm{a}$ & $1.00 \mathrm{a}$ & $60.10 \mathrm{ab}$ & $5.40 \mathrm{c}$ & $10.40 \mathrm{a}$ \\
$\mathrm{Z} 5$ & $1.39 \mathrm{a}$ & $2.50 \mathrm{a}$ & $63.33 \mathrm{a}$ & $81.67 \mathrm{a}$ & $0.33 \mathrm{a}$ & $30.00 \mathrm{a}$ & $1.33 \mathrm{a}$ & $76.00 \mathrm{ab}$ \\
$\mathrm{Z6}$ & $4.18 \mathrm{a}$ & $9.43 \mathrm{ab}$ & $367.50 \mathrm{c}$ & $360.00 \mathrm{bcd}$ & $0.75 \mathrm{a}$ & $67.50 \mathrm{ab}$ & $4.25 \mathrm{abc}$ & $396.00 \mathrm{bc}$ \\
$\mathrm{Z7}$ & $4.17 \mathrm{a}$ & $10.73 \mathrm{ab}$ & $354.67 \mathrm{c}$ & $406.50 \mathrm{~d}$ & $1.00 \mathrm{a}$ & $90.00 \mathrm{~b}$ & $5.67 \mathrm{c}$ & $677.33 \mathrm{c}$ \\
$\mathrm{Z} 8$ & $4.17 \mathrm{a}$ & $9.71 \mathrm{ab}$ & $318.29 \mathrm{c}$ & $394.57 \mathrm{~cd}$ & $1.00 \mathrm{a}$ & $90.00 \mathrm{~b}$ & $5.29 \mathrm{bc}$ & $481.71 \mathrm{c}$ \\
\hline
\end{tabular}

Medias dentro de columnas con letras diferentes son estadísticamente significativas $(\alpha=0.05)$. DAB $=$ diámetro a la altura de la base, Diam 1, 2 = diámetro 1 y 2, RamBas = ramas basales, AngInc = ángulo de inclinación, OrdRam = órdenes de ramificación, NumFru = número de frutos.

Means within columns with different letter are statistically significant $(\alpha=0.05)$. DAB $=$ diameter at the base, Diam 1,2 $=$ diameter 1 and 2, RamBas = basal branches, AngInc $=$ angle of the branches, OrdRam $=$ branching orders, NumFru $=$ number of fruits.

Z3, Z4 y Z5 estuvo relacionada positivamente con la pendiente, y en el caso de la Z1 con la orientación este-oeste; para las primeras tres zonas los individuos juveniles tuvieron menor altura, diámetro basal, cobertura y número de ramas basales. Las Z7 y Z8 fueron sitios poco pedregosos, pero de mayor tamaño de piedras, con suelo relativamente más profundo, que concentraron individuos de mayores dimensiones (Fig. 2).

El ACC de las poblaciones de individuos adultos de $H$. latiflora indicó correlaciones altas para factores ecológicos-variables morfométricas (eje uno $\mathrm{r}=0.97$ y eje dos $\mathrm{r}=0.91$, $\mathrm{p}=0.1400)$. Ambos ejes explicaron el $76.4 \%$ de la varianza total (Cuadro 5). Los resultados indicaron que la profundidad del suelo y la pedregosidad influyeron positivamente en la variación morfométrica (cobertura, número de ramas basales y diámetro basal, además del número de frutos) entre individuos de las $Z 5$, Z6, Z7 y Z8; los individuos de las últimas dos zonas tuvieron mayores dimensiones en estas variables. La pendiente influyó significativamente en la semejanza morfométrica de las poblaciones de las Z3 y Z4, mientras que la variación morfométrica de las Z1 y Z2 estuvo relacionada con la orientación del sitio (Fig. 2). 


\section{CUADRO 5}

Análisis de Correspondencia Canónica (ACC) para ocho poblaciones de juveniles y adultos de Hintonia latiflora y cuatro variables ambientales

TABLE 5

Canonical correspondence analysis (CCA) to eight saplings and adults populations of Hintonia latiflora and four environmental variables

\begin{tabular}{lcccc} 
& \multicolumn{2}{c}{ Juveniles } & \multicolumn{2}{c}{ Adultos } \\
& Eje 1 & Eje 2 & Eje 2 & 0.020 \\
Valores característicos & 0.067 & 0.025 & 0.035 & 0.91 \\
Correlación especies-ambiente & 0.99 & 0.97 & 0.97 & 76.4 \\
Varianza acumulada & 64.7 & 88.7 & 48.7 & 0.517 \\
Correlaciones & & & -0.865 \\
Orientación & & & -0.758 & 0.313 \\
Pendiente & -0.649 & 0.756 & -0.260 & -0.439 \\
Profundidad de suelo & 0.725 & 0.201 & 0.814 & 0.667 \\
Tamaño de piedras & -0.138 & -0.906 & & -0.872 \\
\hline
\end{tabular}

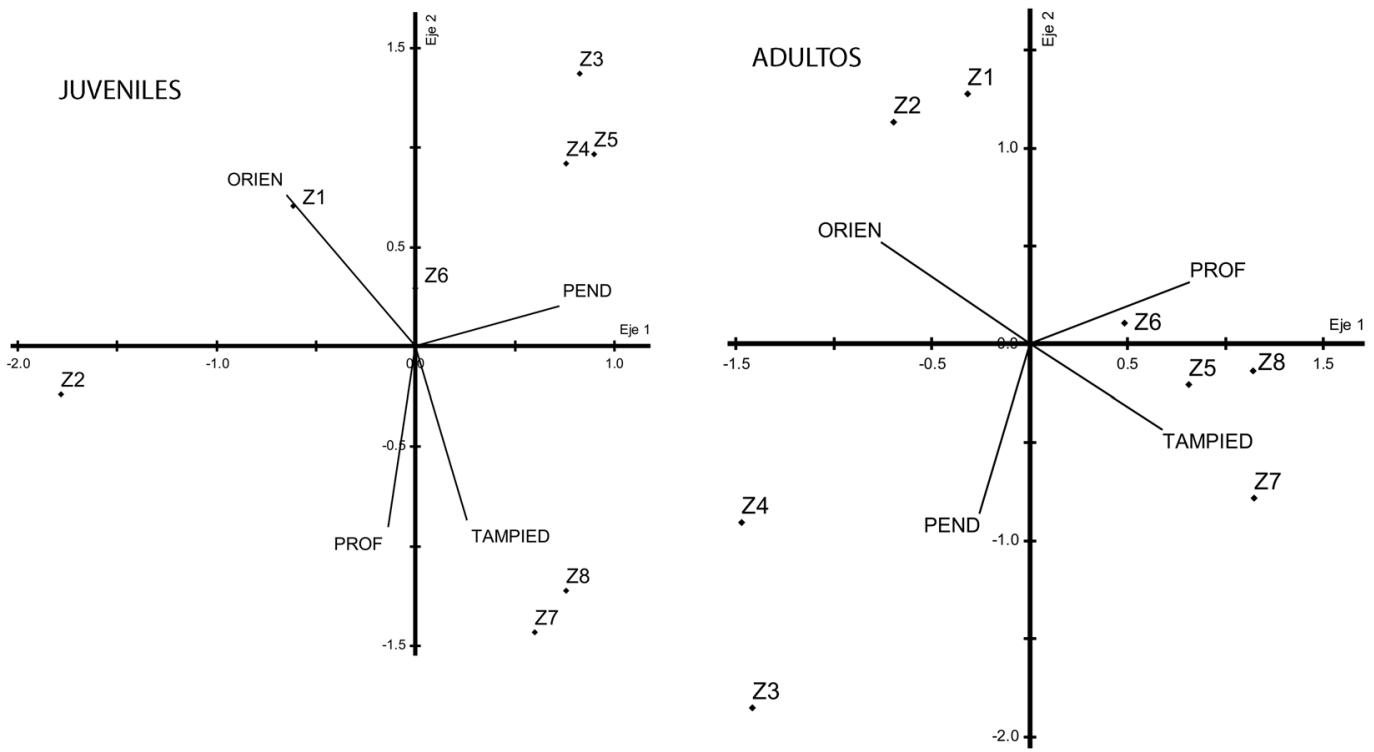

Fig. 2. Diagrama de ordenación del análisis de correspondencia canónica (ACC) que muestra la influencia de los factores ecológicos: orientación del sitio (ORIEN), pendiente (PEND), profundidad del suelo (PROF) y tamaño de piedras (TAMPIE) sobre la arquitectura de individuos juveniles y adultos de $H$. latiflora en las ocho zonas de cosecha. $\mathrm{Z}=$ zona de cosecha. Fig. 2. Ordination diagram of the canonical correspondence analysis (CCA) indicating the influence of the ecological factors: site orientation (ORIEN), slope (PEND), soil depth (PROF), and size of stones (TAMPIE) on the architecture of saplings and adults individuals of Hintonia latiflora in the eight harvesting zones. $\mathrm{Z}=$ harvest zone. 


\section{DISCUSIÓN}

Los individuos descortezados en las zonas de cosecha muestreadas del Alto Balsas fueron principalmente adultos y pocos juveniles, ya que en éstos, la producción y las dimensiones de la corteza requeridas para la cosecha son menores comparadas con los adultos (BeltránRodríguez et al., 2015). Destaca que en las zonas con alta intensidad de cosecha de corteza, se localizan los individuos de ambos estadios de desarrollo con mejores atributos alométricos (altura, diámetro basal y cobertura), así como el mayor número de individuos monopódicos y multirramificados. Este hallazgo advierte que la cosecha de corteza afecta positivamente algunas características morfométricas y reproductivas de la quina amarilla. En un caso similar, Gaoue y Ticktin (2008) demostraron que el descortezamiento no afecta significativamente las tasas y patrones reproductivos en Khaya senegalensis (Desr.) A. Juss., aún cuando se combina con diferentes intensidades de defoliación. Pero otras investigaciones (Botha, Witkowski, \& Shackleton, 2004) evidencian que la cosecha comercial de corteza reduce el diámetro basal y la altura de los tallos de Warburgia salutaris (Bertol.) Chiov., así como la densidad y número de individuos reproductivos en Garcinia lucida Vesque (Guedje, Zuidema, During, Foahom, \& Lejoly, 2007). De manera que la respuesta morfométrica y reproductiva ante la cosecha de corteza, depende tanto de la biología de cada especie como de los factores de sitio (Cunningham et al., 2014).

La densidad promedio de Hintonia latiflora en la región muestreada del Alto Balsas actualmente es baja y dispersa (adultos $=5$ ind.ha ${ }^{-1}$ y juveniles $=6$ ind.ha ${ }^{-1}$ ), como consecuencia de la devastación comercial antes mencionada. En opinión de varios autores (Belloni, Benedetti, \& Scariot, 2007; van Andel \& Havinga, 2008; Huai, Wen, Xu, \& Bai, 2013), la alta intensidad de cosecha de alguna estructura vegetal (en este caso la corteza), generaría como respuesta compensatoria, la elevación de las tasas de repoblación natural, cuestión que estaría por probarse en estas poblaciones de $H$. latiflora. La densidad actual de plántulas de quina amarilla es de 213 ind. ha $^{-1}$ (Beltrán-Rodríguez et al., 2015), pero la sobrevivencia está en constante riesgo con tasas de mortalidad superiores al $95 \%$, debido tanto a procesos de competencia intraespecífica en poblaciones agrupadas, como a restricciones en el desarrollo, por la carencia hídrica del bosque tropical caducifolio.

El análisis de componentes principales indicó que los individuos juveniles y adultos tuvieron en cuatro de las ocho zonas $(\mathrm{Z} 1, \mathrm{Z2}$, Z7 y Z8) mayor cobertura, altura, diámetro basal y ángulo de inclinación de ramas. Solo los adultos de dos de estas zonas (Z1 y Z2) tendieron a ser multirramificados desde la base. Estas poblaciones contrastan morfológicamente con las cuatro restantes, porque presentaron menores dimensiones para los atributos mencionados. La diferencia en tamaños evidenciada entre zonas, tanto en los individuos juveniles como en los adultos, es concluyente de que entre ellos hay diferente producción de corteza.

Con respecto a los factores de sitio, la pendiente, orientación, profundidad del suelo y tamaño de piedras, fueron los elementos del medio que influyeron en la variación en altura, diámetro basal, cobertura y número de ramas basales de $H$. latiflora, y en el caso de los adultos, también el número de frutos. Lo anterior concordó con los resultados obtenidos por Bruschi, Vendramin, Bussotti y Grossoni (2003), quienes señalaron que los factores edáficos $\mathrm{y}$, en su caso, los climáticos, fueron los determinantes de la variación morfológica observada en Quercus petraea (Matt.) Liebl. También, Romero-Méndez, López-Corrujedo, García-de la Peña y Estrada-Rodríguez (2013) han indicado que los morfotipos de Astrophytum myriostigma Lem. (cupuliforme, cónico y deprimido) están correlacionados significativamente con la pendiente y orientación de la ladera; mientras que Ouinsavi y Sokpon (2010) encontraron que la altura total, diámetro de copa y longitud de hojas de $M$. excelsa están altamente correlacionadas con la precipitación y propiedades fisicoquímicas del suelo. 
En las poblaciones de juveniles y adultos de $H$. latiflora de sitios con mayor pendiente se encontraron individuos con menor número de ramas basales y tamaño pequeño, posiblemente por la relación inversa entre pendiente del terreno y retención de nutrimentos del suelo, de acuerdo con la opinión de diversos autores (Chauvel, Lucas, \& Boulet, 1987; Becker, Rabenol, Idol, \& Smith, 1988; González \& Zak, 1996), quienes han confirmado que las especies que habitan en pendientes pronunciadas, responden a un gradiente edáfico en el que predomina menor disponibilidad de agua y nutrimentos y, una textura más arenosa. Debido a ello, los individuos juveniles y adultos de quina amarilla registrados en las Z3, Z4 y Z5 presentan menores dimensiones. Poorter, Jans, Bongers y Van Rompaey (1994) indicaron que, a mayor pendiente, existe una mayor apertura en el dosel y densidad de claros; lo cual concuerda con las características de las zonas de cosecha referidas, con disturbio moderado y soleadas.

Auslander, Nevo e Inbar (2003) y LópezGómez et al. (2012) mostraron que la orientación de la ladera modifica las condiciones microclimáticas de los sitios y esto genera cambios estructurales en las plantas. El ACC anticipa que la orientación este-oeste en juveniles $\left(90^{\circ}-270^{\circ}\right.$; zona 1$)$ y norte-sur en adultos $\left(0^{\circ}-180^{\circ}\right.$; zona 2$)$, está relacionada con individuos de $H$. latiflora generalmente de mayor altura, cobertura extendida y diámetro basal, con tendencia a un mayor número de ramas basales, particularmente en individuos adultos. La orientación sureste y suroeste reciben mayor radiación solar al día (López-Gómez et al., 2012), de manera que los individuos de la Z1 y Z2 son estrictamente heliófitos, como lo indican Huante, Rincón y Acosta (1995) y Velázquez, Cházaro, González y Covarrubias (2011) para poblaciones de $H$. latiflora del occidente de México, y han desarrollado un dosel con una cobertura más extendida. En los resultados de esta investigación, la radiación solar no resultó importante para explicar la arquitectura de individuos juveniles y adultos, aunque sí se aprecia el desarrollo de morfotipos basítonos.
Estos individuos crecieron principalmente en condiciones soleadas, en hábitats con disturbio y apertura del dosel, pero también, se vieron con menor frecuencia en ambientes sombreados asociados a cañadas, lo cual advierte que hay otros factores que también influyen para la multirramificación, aunque no fueron demostrados en este estudio, como el ramoneo, el fuego y la mala práctica de cosecha, que conlleva a la tala basal de la planta; lo que genera el desarrollo de múltiples yemas de crecimiento que con el tiempo se desarrollarán como ramas.

Se aprecia de las Z7 y Z8 que a mayor profundidad del suelo y poca pedregosidad, la quina tiene mayor altura, diámetro basal y cobertura en juveniles y adultos, debido a ello la densidad de individuos con estas dimensiones se resguarda en zonas muy restringidas, dado que de acuerdo con Trejo (1983), los suelos someros y pedregosos dominan en el área de estudio. Es sabido que suelos someros y alta pedregosidad reducen el anclaje de los árboles (caso Nectandra rudis C.K. Allen), los que caen más fácilmente (Cuevas-Guzmán, García-Moya, Vázquez-García, \& Núñez-López, 2008). Un caracter morfológico no estudiado en $H$. latiflora han sido las raíces fasciculadas y extendidas que desarrollan las plantas a menos de $20 \mathrm{~cm}$ de la superficie del suelo, las que le permiten mayor superficie de contacto y posiblemente mayor estabilidad, al compensarse con un mayor diámetro basal para asegurar el adecuado sostén de la cobertura.

Considerando las condiciones edáficas y topográficas documentadas en este estudio, favorables para el desarrollo arbóreo de calidad comercial para cosecha de corteza de $H$. latiflora, se recomienda distribuir plantaciones de quina amarilla en terrenos de poca pendiente (ladera a planicies), escasa pedregosidad (sitios con $<50 \%$ de superficie pedregosa y piedras de $<10 \mathrm{~cm}$ ), suelos profundos $(>10 \mathrm{~cm})$, posición orientada hacia el este-oeste (para favorecer el desarrollo de juveniles-latizales) y sombreados al norte-sur (para posteriormente ubicar adultos-fustales, probablemente dominantes después de que haya ocurrido el aclareo de las poblaciones). De los ocho sitios de muestreo, 
los más recomendables para lograr la talla óptima para fines de extracción comercial de la corteza son el uno, dos, siete y ocho, debido a que favorecen el desarrollo de individuos juveniles y adultos de mayor altura, cobertura y grosor del fuste. En contraste, los sitios tres, cuatro, cinco y seis, tienen pendiente pronunciada, son altamente pedregosos y someros, lo que limita el establecimiento y desarrollo de las plantas de H. latiflora. De hecho, la densidad poblacional promedio en los últimos cuatro sitios es de solo un tercio de lo correspondiente a los primeros indicados, convirtiéndolos en menos productivos para la extracción comercial de corteza.

Las diferencias arquitectónicas representan también un aporte para definir criterios de cosecha y manejo. Las plantas basítonas tienen menor altura y cobertura promedio, y pese a que desarrollan mayor número de ramas basales, el diámetro de éstas y el grosor de la corteza son de menor dimensión en comparación con los árboles mesótonos. La típica arquitectura Leeuwenberg aportaría mayor volumen de corteza por individuo, pero en estado silvestre éstos son los árboles menos abundantes, de ahí que en el área de estudio, los recolectores cosechan por accesibilidad y con mayor frecuencia a individuos arbustivos multirramificados basítonos, es decir, individuos morfométricamente pequeños y muy ramificados. Una vez sometida la zona a plantación forestal, se recomienda que el programa de manejo de $H$. latiflora evite la corta total para mantener el crecimiento monopódico y la mayor producción de cortes longitudinales de corteza. Otra opción es que el manejo en bosques naturales después de la corta total se continúe con la práctica de aprovechamiento en monte bajo con resalvos (es decir, con vástagos rebrotados de los tocones, a lo que se ha llamado en este documento, reiteraciones arquitectónicas); ambos programas de manejo permiten mantener las dos variantes arquitectónicas, reducir costos de inversión por preparación del sitio y conservar in situ a esta especie en la región de estudio. El potencial de esta información se extiende para planificar programas de rehabilitación en áreas afectadas por la explotación comercial, y establecer plantaciones forestales en sitios clave para asegurar el desarrollo de individuos con características deseables para cosecha de corteza, además que se contribuiría a la conservación in situ de H. latiflora en la Cuenca del Alto Balsas.

\section{AGRADECIMIENTOS}

El primer autor agradece el financiamiento otorgado (becario 265032) por el Consejo Nacional de Ciencia y Tecnología (CONACYT) de México y al Colegio de Postgraduados, por los recursos destinados al trabajo de campo. También se agradece a los revisores del documento, que mejoraron sustancialmente la calidad del mismo. Se agradece a las autoridades comunales de Tlalcozotitlán y a Emiliano Soriano, Roberto Flores y Selene Sánchez por su apoyo en el trabajo de campo.

\section{RESUMEN}

Hintonia latiflora es una especie ecológicamente rara y económicamente importante del bosque tropical caducifolio de la Cuenca del Balsas, México, cuya corteza se comercializa como medicinal. Las prácticas de descortezado han modificado la forma de las plantas, su arquitectura y morfometría. Se desconoce, si además, existe influencia de factores topogáficos y edáficos sobre estos atributos. Se propone que las condiciones ecológicas en las áreas de distribución y cosecha determinarán las características morfométricas y la variación de la arquitectura de los individuos. Para probar esta hipótesis, evaluamos la relación de factores topográficos y edáficos en diferentes características morfológicas de juveniles $(\mathrm{n}=143)$ y adultos $(\mathrm{n}=$ 117) de ocho zonas de cosecha de H. latiflora. Las zonas de cosecha, con una superficie de 0.6 ha, fueron seleccionadas al azar con el apoyo de mapas participativos elaborados por los recolectores del área de estudio. Los datos fueron analizados mediante componentes principales (ACP), y correspondencia canónica (ACC). Los primeros tres CP explicaron el $92.1 \%$ de la variación morfológica; la altura, diámetro basal y cobertura fueron las variables que explicaron las diferencias morfométricas entre juveniles y adultos en las ocho zonas de cosecha. La pendiente del terreno, orientación de la pendiente, profundidad del suelo y pedregosidad, fueron los factores ecológicos estadísticamente relacionados con las diferencias morfométricas. Una menor pendiente, poca pedregosidad, profundidad de suelo, orientación E-O en juveniles (azimut $90^{\circ}-270^{\circ}$ ) y N-S en adultos (azimuth $0^{\circ}-180^{\circ}$ ), fueron los factores asociados a mayor 
altura, número de ramas basales, diámetro basal y cobertura en $H$. latiflora. Las zonas con mayor intensidad de cosecha estuvieron integradas por árboles monopódicos y algunos individuos basítonos reiterados. Los adultos de estas zonas tuvieron mayor número de frutos. En estos sitios, las plantas de H. latiflora fueron más abundantes y productivas para corteza, pero la alta intensidad de cosecha influyó en la arquitectura vegetal, formando individuos arbustivos multirramificados que originalmente fueron mesótonos. Las áreas con menor intensidad de cosecha estuvieron caracterizadas por pendientes abruptas, suelos superficiales y pedregosos, dominadas por árboles basítonos de menor talla, delgados y escasos en el área. Estos sitios son los menos recomendados para cosecha, debido a que producen menos corteza por árbol, pero sirven para mantener a la especie en el lugar. Con base en estos resultados, podemos desarrollar programas de rehabilitación en áreas afectadas por la explotación comercial, establecer plantaciones forestales en sitios clave para asegurar el desarrollo de individuos con características deseables para cosecha de corteza y contribuir a la conservación in situ de H. latiflora en la Cuenca del Alto Balsas.

Palabras clave: arquitectura vegetal, bosque tropical caducifolio, corteza medicinal, cosecha comercial, variación morfométrica.

\section{REFERENCIAS}

Alexiades, M. \& Shanley, P. (2004). Productos Forestales, Medios de Subsistencia y Conservación. Estudios de Caso sobre Sistemas de Manejo y Productos Forestales No Maderables. Volumen 3-América Latina. (1st ed.). Indonesia: Centro para la Investigación Forestal Internacional (CIFOR)

Auslander, M., Nevo, E., \& Inbar, M. (2003). The effects of slope orientation on plant growth, developmental instability and susceptibility to herbivores. Journal of Arid Environments, 55(3), 405-416.

Barthélémy, D., \& Caraglio, Y. (2007). Plant architecture: a dynamic, multilevel and comprehensive approach to plant form, structure and ontogeny. Annals of Botany, 99, 375-407.

Becker, P., Rabenold, P., Idol, J., \& Smith, A. (1988). Water potential gradients for gaps and slopes in a Panamanian tropical moist forest's dry season. Journal of Tropical Ecology, 4(2), 173-184.

Beltrán-Rodríguez, L., Romero-Manzanares, A., LunaCavazos, M., Vibrans, H., Manzo-Ramos, F., CuevasSánchez, J., \& García-Moya, E. (2015). Historia natural y cosecha de corteza de quina amarilla Hintonia latiflora (Rubiaceae). Botanical Sciences, 93(2), 1-12.

Belloni, I., Benedetti, I., \& Scariot, A. (2007). Ethnobotany and effects of harvesting on the population ecology of Syngonanthus nitens (Bong.) Ruhland (Eriocaulaceae), a NTFP from Jalapao Region, Central Brazil. Economic Botany, 6(1), 73-85.

Botha, J., Witkowski, E., \& Shackleton, C. (2004). The impact of commercial harvesting on Warburgia salutaris ("pepper-bark tree") in Mpumalanga, South Africa. Biodiversity and Conservation, 13, 1675-1698.

Bruschi, P., Vendramin, G., Bussotti, F., \& Grossoni, P. (2003). Morphological and molecular diversity among Italian populations of Quercus petraea (Fagaceae). Annals of Botany, 91(6), 707-716.

Chauvel, A., Lucas, Y., \& Boulet, R. (1987). On the genesis of the soil mantle of the region of Manaus, Central Amazonia, Brazil. Experientia, 43(3), 234-241.

Cuevas-Guzmán, R., García-Moya, E., Vázquez-García, J., \& Núñez-López, N. (2008). Estructura poblacional y relaciones ambientales del árbol tropical Nectandra rudis (Lauraceae), una especie rara en el occidente de México. Revista Biología Tropical, 56(1), 247-256.

Cunningham, A., Campbell, B., \& Luckert, M. (2014). Bark. Use, Management, and Commerce in Africa. Advances in Economic Botany, 17, 1-288.

Di Rienzo, J., Casanoves, F., Balzarini, M., Gonzalez, L., Tablada, M., \& Robledo, C. (2013). InfoStat (Versión 2013). Grupo InfoStat, FCA, Universidad Nacional de Córdoba, Argentina.

Gaoue, O., \& Ticktin, T. (2008). Impacts of bark and foliage Harvest on Khaya senegalensis (Meliaceae) reproductive performance in Benin. Journal of Applied Ecology, 45, 34-40.

García, E. (1988). Modificaciones al sistema de clasificación climática de Köppen (para adaptarlo a las condiciones de la República Mexicana) (2nd ed.). México: Universidad Nacional Autónoma de México, Instituto de Geografía.

González, O., \& Zak, D. (1996). Tropical dry forest of St. Lucia, West Indies: Vegetation and soil properties. Biotropica, 28, 618-626.

Guedje, N., Zuidema, P., During, H., Foahom, B., \& Lejoly, J. (2007). Tree bark as a non-timber forest product: The effect of bark collection on population structure and dynamics of Garcinia lucida Vesque. Forest Ecology and Management, 240, 1-12.

Hallé, F. (2010). Arquitectura de los Árboles. Boletín de la Sociedad Argentina de Botánica, 45(3-4), 405-418.

Hallé, F., Oldemann, R. A. A., \& Tomlinson, P. (1978). Tropical trees and forest: an architectural analysis. Berlin: Springer-Verlag.

Hernández-Apolinar, M., Valverde, T., \& Purata, S. (2006). Demography of Bursera glabrifolia, a tropical tree 
used for folk woodcrafting in Southern Mexico: An evaluation of its management plan. Forest Ecology and Management, 223(1), 139-151.

Hersch-Martínez, P. (2007). La industrialización químicofarmacéutica mexicana y la flora: el caso de los Laboratorios Garcol. Boletín de la Sociedad Química de México, 1, 107-114.

Hersch-Martínez, P. \& Fierro, A. (2001). El comercio de plantas medicinales: algunos rasgos significativos en el centro de México. In B. Rendón, S. Rebollar, J. Caballero, \& M. Martínez-Alfaro, Plantas, Cultura y Sociedad. Estudio sobre la relación entre los seres humanos y las plantas de los albores del siglo XXI (1st ed., pp. 53-75). Mexico City: UAM, SEMARNAT.

Hersch-Martínez, P., Fierro, A., Guerrero, C., \& Pérez, A. (2000). Tendencias en la progresión de la colecta de algunas especies medicinales silvestres de relevancia comercial en la colindancia de Puebla y Guerrero, México. In R. Monroy, H. Colín, \& C. Boyas, Los sistemas agroforestales de Latinoamérica y la selva baja caducifolia en México (1st ed., pp. 573-580). Cuernavaca, Morelos: IICA, INIFAP, UAEMor.

Huai, H., Wen, G., Xu, W., \& Bai, W. (2013). Effects of commercial harvesting on population characteristics and rhizome yield of Anemone altaica. Economic Botany, 67(1), 41-50.

Huante, P., Rincón, E., \& Acosta, I. (1995). Nutrient availability and growth rate of 34 woody species from a tropical deciduous forest in Mexico. Functional Ecology, 9, 849-858.

INEGI. (2009). Prontuario de información geográfica municipal de los Estados Unidos Mexicanos. Copali1lo, Guerrero, México.

Interián-Ku, V., Valdez-Hernández, J., García-Moya, E., Romero-Manzanares, A., Borja, M., \& VaqueraHuerta, H. (2009). Arquitectura y morfometría de dos especies arbóreas en una selva baja caducifolia del sur de Yucatán, México. Boletín de la Sociedad Botánica de México, 85, 17-29.

López-Gómez, V., Zedillo-Avelleyra, P., Anaya-Hong, S., González-Lozada, E., \& Cano-Santana, Z. (2012). Efecto de la orientación de la ladera sobre la estructura poblacional y ecomorfología de Neobuxbaumia tetetzo (Cactaceae). Botanical Sciences, 90, 453-457.

Lorence, D., \& Taylor, C. (2011). Rubiaceae. Flora Mesoamericana, 4, 230-232.

Martínez-Sánchez, J., Meave, J., \& Bongers, F. (2008). Light-related variation in sapling architecture of three shade-tolerant tree species of the Mexican rain forest Revista Chilena de Historia Natural, 81, 361-371.
Mata, R., Navarrete, A., Cristians, S., Hersch-Martínez, P., \& Bye, R. (2009). Plantas Medicinales de México. Monografia Cientifica. Pruebas de Control de Calidad (Identificación y Composición), Eficacia y Seguridad. Copalchi - Hintonia latiflora (Sessé et Mociño ex DC.) Bullock (Rubiaceae) (1 ${ }^{\mathrm{a}}$. ed.). D.F., México: Sentido Giratorio Ediciones.

McCune, B., \& Mefford, M. (2011). PC-ORD. Multivariate analysis of ecological data (Version 6). Gleneden Beach, Oregon, U.S.A.: MjM Software Design.

Ochoterena, H. (2000). Systematics of Hintonia Bullock and the Portlandia complex (Rubiaceae). (PhD dissertation). Cornell University, Ithaca, N.Y., USA.

Oke, T. (1987). Boundary Layer Climates (2nd ed.). Taylor \& Francis, Routledge

Ouinsavi, C., \& Sokpon, N. (2010). Morphological variation and ecological structure of Iroko (Milicia excelsa Welw. CC Berg) populations across different biogeographical zones in Benin. International Journal of Forestry Research, 10, 1-10.

Pearcy, R., Muraoka, H., \& Valladares, F. (2005). Crown architecture in sun and shade environments: assessing function and trade-offs with a three-dimensional simulation model. New Phytologist, 166(3), 791-800.

Pineda-Herrera, E., Valdez-Hernández, J., López-López, M., Salgado-Ugarte, I., \& Manzano-Méndez, F. (2011). Arquitectura de dos especies arbóreas de una Selva Alta Perennifolia de Oaxaca. In A. Endara-Agramont, A. Mora-Santacruz, \& J. Valdez-Hernández, Bosques y Árboles del Trópico Mexicano: Estructura, Crecimiento y Usos. (1 st ed., pp. 23-36). Jalisco, México: Universidad de Guadalajara. CUCBA.

Poorter, L., Bongers, L., \& Bongers, F. (2006). Architecture of 54 moist-forest tree species: traits, trade-offs, and functional groups. Ecology, 87(5), 1289-1301.

Poorter, L., Jans, L., Bongers, F., \& Van Rompaey, R. (1994). Spatial distribution of gaps along three catenas in the moist forest of Tai National Park, Ivory Coast. Journal of Tropical Ecology, 10(03), 385-398.

Rendón-Carmona, H., Martínez-Yrízar, A., Balvanera, P., \& Pérez-Salicrup, D. (2009). Selective cutting of woody species in a Mexican tropical dry forest: Incompatibility between use and conservation. Forest Ecology and Management, 257(2), 567-579.

Romero-Méndez, U., López-Corrujedo, H., García-de la Peña, C., \& Estrada-Rodríguez, J. (2013).Variación ecomorfológica de Astrophytum myriostigma (Caryophylalles: Cactaceae) en una población de la sierra 
El Sarnoso, Durango, México. Revista Chilena de Historia Natural, 86, 357-364.

Stanley, E., Voeks, R., \& Short, L. (2012). Is non-timber forest product harvest sustainable in the less developed world? A Systematic Review of the Recent Economic and Ecological Literature. Ethnobiology and Conservation, 1(9), 1-39.

Trejo, I. (1983). Estudio de Vegetación en la Zona de Tlalcozotitlán en la Depresión Oriental del Rio Balsas, Gro (Tesis de Maestría). Universidad Nacional Autónoma de México, Mexico. van Andel, T., \& Havinga, R. (2008). Sustainability aspects of commercial medicinal plant harvesting in Suriname. Forest Ecology and Management, $256,1540-1545$

Velázquez, R., Cházaro, M., González, R., \& Covarrubias, H. (2011). Árboles de las Barrancas de los ríos Santiago y Verde (1 ${ }^{\text {a }}$. ed.). Jalisco, México: Comisión Estatal del Agua de Jalisco.

Vester, H. F. M. (2002). Modelos arquitectónicos en la flora arbórea de Yucatán. Boletín de la Sociedad Botánica de México, 71, 45-57. 